\title{
APPLICATION OF MUSIC TO ARRAYS WITH MULTIPLE INVARIANCES
}

\author{
A. Lee Swindlehurst* ${ }^{*}$ Petre Stoica ${ }^{\dagger}$, Magnus Jansson ${ }^{\ddagger}$ \\ ${ }^{*}$ Dept. of Electrical \& Computer Engineering, Brigham Young University, Provo, Utah, USA \\ ${ }^{\dagger}$ Dept. of Systems \& Control, Uppsala University, Uppsala, SWEDEN \\ †Dept. of Signals, Sensors, \& Systems, Royal Institute of Technology, Stockholm, SWEDEN \\ email: swindle@ee.byu.edu, ps@syscon.uu.se, magnusj@s3.kth.se
}

\begin{abstract}
This paper describes generalizations of the MUSIC and root-MUSIC algorithms for direction of arrival (DOA) estimation to arrays composed of multiple translated subarrays. The advantage of these new approaches is that the DOAs can be estimated using either a one-dimensional search or by rooting a polynomial, as opposed to a multidimensional search as required by the Multiple Invariance (MI)-ESPRIT algorithm. While MI-MUSIC and root-MI-MUSIC are not statistically efficient like MI-ESPRIT, they do perform better than a single invariance implementation of ESPRIT, and are thus better suited for finding the initial conditions required by the MI-ESPRIT search.
\end{abstract}

\section{INTRODUCTION}

The elements of a sensor array are often arranged in a very regular geometry. Linear, circular, and rectangular arrays are common examples. These special geometries can be exploited in developing computationally efficient algorithms for direction of arrival (DOA) estimation. Root-MUSIC [1], IQML [2], and MODE [3] are well-known examples that take advantage of the structure of uniform linear arrays (ULAs) to obtain DOA estimates via polynomial rooting. ESPRIT [4] is an alternative that only requires an array composed of two identical, translated subarrays to achieve rooting-based DOA estimates. Similar techniques have been developed for circular arrays [5] and for rectangular arrays where both azimuth and elevation angles must be determined $[6,7,8,9]$.

Of particular relevance to this paper is the so-called Multiple Invariance (MI)-ESPRIT algorithm [10], which generalizes ESPRIT to handle arrays composed of multiple identical subarrays. While MI-ESPRIT is known to be asymptotically statistically efficient for its given set of assumptions (provided that the subarrays do not share elements), it requires a multidimensional search for the DOA parameters. ESPRIT can always be applied to a multiple invariance array to obtain initial estimates for the search, but in doing so it may not be able to use all of the array elements nor all available structure.

In this paper, we generalize the well-known MUSIC algorithm to handle arrays with multiple translated subarrays. Unlike MI-ESPRIT, the new algorithm estimates the DOAs using only a one-dimensional search, and unlike standard ESPRIT it is able to enforce the constraint that the subarray responses for a given source are related by a constant on the unit circle. Although the proposed MI-MUSIC algorithm does not share the statistical optimality of MIESPRIT, it provides more accurate initial estimates than regular ESPRIT since it is always able to exploit all array invariances and enforce the aforementioned unit circle constraint. We also show how the root-MUSIC technique can be extended to MI arrays. The root-MUSIC approach is attractive since it eliminates the need for even a onedimensional search for the DOA parameters. While the standard version of root-MUSIC finds the zeros of a scalar polynomial to estimate the DOAs, the MI version requires calculation of the zeros of a matrix polynomial. The asymptotic performance of root-MI-MUSIC will be equivalent to that of MI-MUSIC, and hence is suboptimal.

\section{MATHEMATICAL BACKGROUND}

We assume an array composed of $M$ sensors that receives the signals from $d<M$ sources. The array output is denoted by the $M \times 1$ vector $\mathbf{x}(t)$, and satisfies

$$
\mathbf{x}(t)=\mathbf{F}(\boldsymbol{\theta}) \mathbf{s}(t)+\mathbf{n}(t)
$$

where $\theta=\left[\theta_{1}, \cdots, \theta_{d}\right]^{T}$ is the DOA vector, $\mathrm{s}(t)$ the $d \times 1$ vector of signal waveforms, $\mathbf{n}(t)$ the $M \times 1$ vector of noise and interference, and $\mathbf{F}(\boldsymbol{\theta})=\left[\mathbf{f}\left(\theta_{1}\right) \cdots \mathbf{f}\left(\theta_{d}\right)\right]$ is the $M \times d$ matrix whose columns are the array response vectors for each source. We assume that there are no coherent signals and that the noise is spatially white ${ }^{1}: \mathcal{E}\left\{\mathbf{n}(t) \mathbf{n}^{*}(t)\right\}=\sigma^{2} \mathbf{I}$. Thus, the eigenvectors $\mathbf{E}_{s}$ associated with the $d$ largest eigenvalues of the array covariance matrix $\mathbf{R}_{x x}$ span the same space as the columns of $\mathbf{F}(\boldsymbol{\theta})$. With noisy data, the signal and noise subspaces must be approximated using the sample covariance matrix $\hat{\mathbf{R}}_{x x}$, and we denote the matrix made from the eigenvectors associated with the $d$ largest eigenvalues by $\hat{\mathbf{E}}_{s}$.

The sensor array is assumed to be composed of $p$ identical subarrays of $m$ elements each, the subarrays being displaced at arbitrary but known distances along a straight line. In general, $m p \geq M$ since different subarrays may have elements in common. Examples of arrays that satisfy the above criteria can be found in [10]. One subarray is chosen as the reference, and the known displacement of

\footnotetext{
${ }^{1}$ In our notation, the symbol $(\cdot)^{*}$ denotes the complex conjugate transpose operation.
} 
the other subarrays from the reference is denoted by $\delta_{k}$, for $k=1, \cdots, p-1$. The structure of this type of array can be described mathematically by the following equation:

$$
\mathbf{J F}(\boldsymbol{\theta}) \stackrel{\text { def }}{=} \tilde{\mathbf{F}}=\left[\begin{array}{c}
\mathbf{A} \\
\mathbf{A} \Phi^{\delta_{1}} \\
\vdots \\
\mathbf{A} \Phi^{\delta_{p-1}}
\end{array}\right]
$$

where $\mathbf{J}$ is a known $m p \times M$ selection matrix, $\mathbf{A}$ represents the unknown response of the reference subarray, and $\boldsymbol{\Phi}$ is a diagonal matrix defined by

$$
\mathbf{\Phi}=\operatorname{diag}\left\{e^{j 2 \pi \sin \left(\theta_{1}\right) / \lambda}, \cdots, e^{j 2 \pi \sin \left(\theta_{d}\right) / \lambda}\right\},
$$

where $\lambda$ is the wavelength of the received signals.

The dependence of the model on $\theta$ is confined to the unit modulus diagonal elements of $\boldsymbol{\Phi}$. The selection matrix $\mathbf{J}$ is needed to handle overlapping subarrays; if no elements are shared between subarrays, then the rows of $\mathbf{F}(\theta)$ can be arranged so that $\mathbf{J}=\mathbf{I}$. We can also write (2) column-wise as follows:

$$
\mathbf{J f}\left(\theta_{k}\right)=\boldsymbol{\rho}\left(\theta_{k}\right) \otimes \mathbf{a}_{k},
$$

where $\otimes$ denotes the Kronecker product, $\mathbf{a}_{k}$ is the $k^{\text {th }}$ column of $\mathbf{A}$, and

$$
\rho\left(\theta_{k}\right)=\left[\begin{array}{llll}
1 & e^{j 2 \pi \delta_{1} \sin \left(\theta_{k}\right) / \lambda} & \cdots & e^{j 2 \pi \delta_{p-1} \sin \left(\theta_{k}\right) / \lambda}
\end{array}\right]^{T} .
$$

In [10], equation (2) is exploited by minimizing the following weighted least squares criterion, referred to as MIESPRIT:

$$
\hat{\mathbf{A}}, \hat{\boldsymbol{\theta}}, \hat{\mathbf{T}}=\arg \min _{A, \theta, T}\left\|\mathbf{J} \hat{\mathbf{E}}_{s} \mathbf{W}^{1 / 2}-\tilde{\mathbf{F}} \mathbf{T}\right\|_{F}^{2} .
$$

When there are no overlapping subarrays, this algorithm has been shown to be asymptotically statistically efficient for a suitable choice of the weighting matrix $\mathbf{W}$. However, implementing (6) requires a search over $\theta$ and the elements of either $\mathbf{A}$ or $\mathbf{T}$, whichever is of smaller dimension. While the standard single-invariance ESPRIT algorithm can be used to obtain an initial estimate of the desired parameters, the need for a multidimensional search of relatively high dimension is a significant drawback. In the next section we present a new algorithm that (unlike ESPRIT) exploits the full invariance structure of the array, and (unlike MIESPRIT) only requires a one-dimensional search.

\section{MULTIPLE INVARIANCE MUSIC}

The key idea behind the approach presented in this section is a modification of the criterion in (6) that places the subspace fitting matrix $T$ on the left rather than right hand term (in which case $W$ in (6) is irrelevant):

$$
\hat{\mathbf{A}}, \hat{\boldsymbol{\theta}}, \hat{\mathbf{T}}=\arg \min _{A, \theta, T}\left\|\mathbf{J} \hat{\mathbf{E}}_{s} \mathbf{T}-\tilde{\mathbf{F}}\right\|_{F}^{2} .
$$

This has the advantage of making the error term linear in both $\mathbf{A}$ and $\mathbf{T}$, so that estimates of both can be obtained in closed form. The drawback is that an unknown coloring of the statistics of the error term is introduced. While a weighting matrix could be used to optimally account for this color, the resulting solution would still require a multidimensional search over the $d$ DOAs. In the interest of finding a simpler solution, we prefer to focus on the unweighted criterion of $(7)$.

Minimization of the criterion in (7) with respect to $\mathbf{T}$ yields the following concentrated problem:

$$
\hat{\mathbf{A}}, \hat{\boldsymbol{\theta}}=\arg \min _{A, \theta} \operatorname{Tr}\left(\tilde{\mathbf{F}}^{*} \boldsymbol{\Pi}_{J}^{\perp} \hat{E}_{s} \tilde{\mathbf{F}}\right),
$$

where $\boldsymbol{\Pi}_{J \hat{E}_{s}}^{\perp}=\mathbf{I}-\mathbf{J} \hat{\mathbf{E}}_{s}\left(\hat{\mathbf{E}}_{s}^{*} \mathbf{J}^{T} \mathbf{J} \hat{\mathbf{E}}_{s}\right)^{-1} \hat{\mathbf{E}}_{s}^{*} \mathbf{J}^{T}$. Using (4), the minimization of (8) becomes

$$
\min \sum_{k=1}^{d}\left(\rho\left(\theta_{k}\right) \otimes \mathbf{a}_{k}\right)^{*} \mathbf{\Pi}_{J \hat{E}_{s}}^{\perp}\left(\boldsymbol{\rho}\left(\theta_{k}\right) \otimes \mathbf{a}_{k}\right) .
$$

Since each term in the sum depends only on the parameters of one source $\left(\theta_{k}\right.$ and $\left.\mathbf{a}_{k}\right)$, the minimization can be performed for one source at a time by finding the $d$ deepest minima of the criterion

$$
V(\theta, \mathbf{a})=(\boldsymbol{\rho}(\theta) \otimes \mathbf{a})^{*} \boldsymbol{\Pi}_{J \hat{E}_{s}}^{\perp}(\boldsymbol{\rho}(\theta) \otimes \mathbf{a}) .
$$

Minimizing (10) amounts to finding array response vectors $\boldsymbol{\rho}(\theta) \otimes$ a that are as "close" as possible to the multipleinvariance signal subspace defined by $\mathbf{J} \hat{\mathbf{E}}_{s}$. This is the basic idea behind the MUSIC algorithm, and consequently we refer to this approach as Multiple Invariance (MI)-MUSIC.

For convenience, we will rewrite (10) as follows

$$
\begin{aligned}
V(\theta, \mathbf{a}) & =\mathbf{a}^{*}(\rho(\theta) \otimes \mathbf{I})^{*} \Pi_{J \hat{E}_{s}}^{\perp}(\rho(\theta) \otimes \mathbf{I}) \mathbf{a} \\
& =\mathbf{a}^{*} \mathbf{P}(\theta) \mathbf{a},
\end{aligned}
$$

where $\mathbf{P}(\theta)$ is defined in an obvious way. Since (12) is quadratic in $\mathbf{a}$, we can explicitly minimize it with respect to a provided that a constraint is employed to eliminate the trivial $\hat{\mathbf{a}}=0$ solution. We present the solution below for the quadratic constraint $\mathbf{a}^{*} \mathbf{a}=1$. Minimizing (12) subject to $\mathbf{a}^{*} \mathbf{a}=1$ yields the following solution in terms of the eigenvalue decomposition of $\mathbf{P}(\theta)$ :

$$
\hat{\theta}=\arg \min _{\theta} \lambda_{\min }(\mathbf{P}(\theta)),
$$

where $\lambda_{\min }(\cdot)$ is the minimum eigenvalue of its matrix argument. The subarray response vector â associated with $\hat{\theta}$ is taken to be the eigenvector associated with $\lambda_{\min }(\mathbf{P}(\hat{\theta}))$. It is easy to see that the above implementation reduces to the standard MUSIC algorithm in the limit where each subarray consists of a single array element.

\section{MULTIPLE INVARIANCE ROOT-MUSIC}

The root-MUSIC algorithm [1] was developed as a specialization of MUSIC to uniform linear arrays. It takes advantage of the resulting Vandermonde structure in the steering vectors to write the MUSIC criterion as a polynomial, $d$ of whose roots will ideally lie on the unit circle. The phase of these complex roots determines the DOAs. In the presence 
of noise, the $d$ roots closest to the unit circle are chosen for use in estimating the DOAs. In this section, we show how the root-MUSIC idea is extended to MI arrays. We must assume that the subarray displacements are rational multiples of one another; in other words, there must exist integers $\ell$ and $n$ satisfying $\delta_{q}=(\ell / n) \delta_{r}$ for all $q, r=1, \cdots, p-1$. To simplify the discussion that follows, we will assume that the displacements are integer multiples of $\lambda / 2$ (and hence each $\delta_{k}$ is an integer), although this is not strictly necessary.

With the above assumptions, we may write (5) as the following polynomial vector:

$$
\rho(z)=\left[\begin{array}{llll}
1 & z^{\delta_{1}} & \cdots & z^{\delta_{p-1}}
\end{array}\right]^{T}
$$

where $z=e^{j \pi \sin (\theta)}$. The criterion in (12) then becomes

$$
V(z, \mathbf{a})=\mathbf{a}^{*} \mathbf{P}(z) \mathbf{a},
$$

where

$$
\begin{aligned}
\mathbf{P}(z) & =(\boldsymbol{\rho}(z) \otimes \mathbf{I})^{*} \mathbf{\Pi}_{J \hat{E}_{s}}^{\perp}(\boldsymbol{\rho}(z) \otimes \mathbf{I}) \\
& =\sum_{k=0}^{p-1} \sum_{n=0}^{p-1} z^{* \delta_{k}} z^{\delta_{n}}\left(\mathbf{\Pi}_{J \hat{E}_{s}}^{\perp}\right)_{k n}
\end{aligned}
$$

and $\delta_{0} \stackrel{\text { def }}{=} 0$. The matrix $\left(\Pi_{J \hat{E}_{s}}^{\perp}\right)_{k n}$ is the $m \times m$ sub-block of the $m p \times m p$ matrix $\Pi_{J \hat{E}_{s}}^{\perp}$ located at block row $k+1$ and block column $n+1$. In the absence of noise, if we pick a value of $z$ corresponding to one of the DOAs, then there will exist a vector a such that $V(z, \mathbf{a})=0$. In other words, we are looking for values of $z$ where the matrix $\mathbf{P}(z)$ drops rank. The process of finding such $z$ 's is complicated by the presence of the conjugate powers of $z$ in (17). To overcome this problem, we observe that since the desired values of $z$ lie on the unit circle, $z^{*}=z^{-1}$, and we may instead consider the following matrix polynomial:

$$
\tilde{\mathbf{P}}(z)=\sum_{k=0}^{p-1} \sum_{n=0}^{p-1} z^{\delta_{n}-\delta_{k}}\left(\Pi_{J \hat{E}_{s}}^{\perp}\right)_{k n} .
$$

Thus, to estimate the DOAs, we seek the roots of the matrix polynomial $\tilde{\mathbf{P}}(z)$, or the solutions to

$$
\operatorname{det} \tilde{\mathbf{P}}(z)=0 \text {. }
$$

To find the subarray steering vector associated with a particular root $\hat{z}$, we solve the linear equation $\tilde{\mathbf{P}}(\hat{z}) \mathbf{a}=0$.

The process described above is entirely analogous to the standard root-MUSIC algorithm; in fact, if we specialize to the case of a uniform linear array with $\delta_{k}=k, m=1$, and $p=M$, then $\tilde{\mathbf{P}}(z)$ is equal to the scalar polynomial used by root-MUSIC. As with root-MUSIC, the order of the polynomial det $\tilde{\mathbf{P}}(z)$ is larger than $d$, and some method is needed to separate the desired roots from the extraneous ones. In the presence of noise, this is done by picking the $d$ roots closest to the unit circle.

\section{SIMULATIONS}

The output of the eight-element array depicted in Figure 1 was simulated to compare the performance of MI-MUSIC

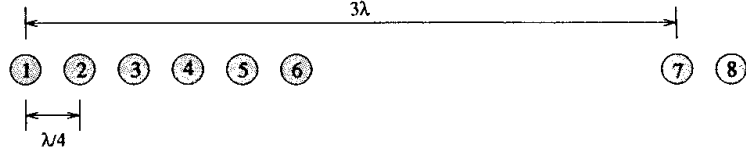

Figure 1: Array used for the simulation examples.

and MI-root-MUSIC with ESPRIT, MI-ESPRIT, and the alternating least-squares (ALS) method of [11]. Each of the first six elements are separated by $\lambda / 4$, while elements 7 and 8 are separated from the first group by $3 \lambda$. The array is grouped into four subarrays of two elements each; subarray $k$ consists of elements $2 * k-1$ and $2 * k$. Under these assumptions, the steering vector matrix is given by

$$
\tilde{\mathbf{F}}=\left[\begin{array}{c}
\mathbf{A} \\
\mathbf{A} \boldsymbol{\Phi}^{\lambda / 2} \\
\mathbf{A} \boldsymbol{\Phi}^{\lambda} \\
\mathbf{A} \boldsymbol{\Phi}^{3 \lambda}
\end{array}\right]
$$

The ALS algorithm attempts a least-squares fit of the data to the following steering vector matrix model:

$$
\tilde{\mathbf{F}}=\left[\begin{array}{c}
\mathbf{A} \Phi_{1} \\
\mathbf{A} \Phi_{2} \\
\mathbf{A} \Phi_{3} \\
\mathbf{A} \Phi_{4}
\end{array}\right]
$$

and thus in its initial stage does not exploit knowledge of the subarray displacements. Consequently, the ALS estimates of $\Phi_{1}, \cdots, \Phi_{4}$ are unstructured, and care must be taken in deriving estimates of the DOAs from them. In the simulations described below, the diagonal elements of $\hat{\mathbf{\Phi}}_{4} \hat{\mathbf{\Phi}}_{1}^{-1}$ were used to determine the DOA estimates, as this approach provided better performance than using either $\hat{\mathbf{\Phi}}_{2} \hat{\mathbf{\Phi}}_{1}^{-1}$ or $\hat{\boldsymbol{\Phi}}_{3} \hat{\boldsymbol{\Phi}}_{1}^{-1}$ alone, or averaging the estimates obtained from all three. Note that there is an inherent ambiguity in using $\hat{\mathbf{\Phi}}_{4} \hat{\boldsymbol{\Phi}}_{1}^{-1}$ to estimate the DOAs, since subarrays 1 and 4 are separated by $3 \lambda$. For these simulations, the sources were assumed to be close enough to broadside so that this ambiguity could be resolved. ESPRIT was implemented using subarrays 1 and 4 , and the resulting ambiguity was handled in the same way. Providing ALS and ESPRIT with the information necessary to resolve the DOA ambiguity gives them a significant performance advantage. If these algorithms had to base their DOA estimates on more closely spaced subarrays (such as subarrays 1 and 2, using the matrix $\boldsymbol{\Phi}_{2} \boldsymbol{\Phi}_{1}^{-1}$ ), their performance would be much poorer. Since the proposed MUSIC algorithms exploit the full structure of the steering vector matrix, there is no DOA ambiguity, and no prior information about the location of the sources is needed or used by the algorithms.

In each of the simulation examples outlined below, two sources were present, 100 samples of data were taken from the array, ESPRIT was used to initialize the MI-ESPRIT and ALS iterations, and algorithm performance for each case was calculated based on an average over 1000 independent Monte Carlo trials. In no case were the simulated scenarios difficult enough to cause MI-MUSIC to be unable to resolve the source DOAs. 


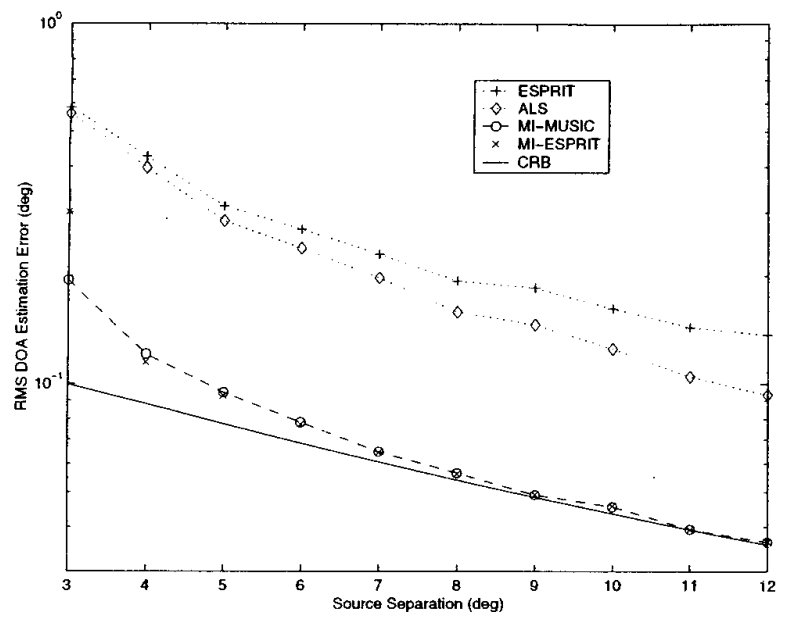

Figure 2: Performance of ESPRIT, ALS, MI-MUSIC, and MI-ESPRIT as a function of source separation.

Performance versus Angular Separation - Two uncorrelated sources with SNRs of $10 \mathrm{~dB}$ were simulated, with the DOA of one fixed at $5^{\circ}$, and the other varied between $2^{\circ}$ and $-7^{\circ}$. The root mean square (RMS) DOA estimation error of the algorithms was calculated at the various angular separations, and is plotted in Figure 2 together with the Cramér-Rao bound (CRB) for the problem. Results for MI-root-MUSIC are not plotted because they were nearly the same as for MI-MUSIC. The one exception was at the smallest angular separation, where MI-root-MUSIC had a somewhat lower RMS error of 0.16. For separations beyond about $6^{\circ}$, both MI-ESPRIT and MI-MUSIC achieve the CRB. This is not surprising since MUSIC has been shown to be asymptotically efficient for uncorrelated sources. In the toughest case (source separation of only $3^{\circ}$ ), MI-MUSIC has a notable advantage over MI-ESPRIT. Both ALS and ESPRIT are significantly above the bound.

Performance versus Correlation Coefficient - The advantage of MI-ESPRIT becomes apparent as the sources become correlated. This example is similar to the first two, except that the two sources are fixed at $\pm 5^{\circ}$ with $20 \mathrm{~dB}$ SNR, and the magnitude of the correlation coefficient is varied between 0 and 1 (the phase of the correlation coefficient was assumed to be zero in all cases). The results are displayed in Figure 3. The performance of MI-ESPRIT is insensitive to correlation, and achieves the CRB in all cases. The other algorithms begin to degrade when the sources are more than about $50 \%$ correlated, but the performance ordering observed in the first two examples is still preserved. As in the previous example, the performance of MI-root-MUSIC is essentially identical to MI-MUSIC.

\section{REFERENCES}

[1] A. J. Barabell, "Improving the Resolution Performance of Eigenstructure-Based Direction-Finding Al-

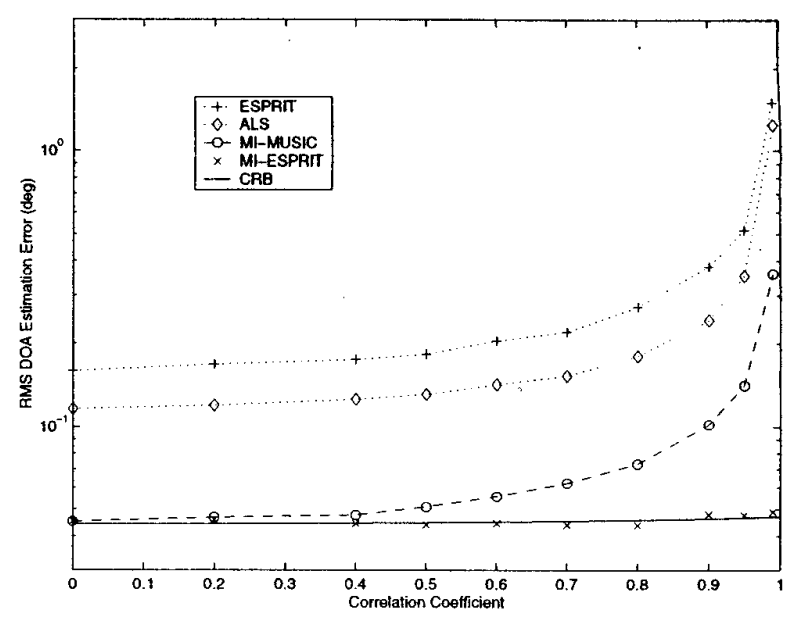

Figure 3: Performance of ESPRIT, ALS, MI-MUSIC, and MI-ESPRIT versus the correlation coefficient magnitude.

gorithms", In Proc. IEEE ICASSP, pages 336-339, 1983.

[2] Y. Bresler and A. Macovski, "Exact Maximum Likelihood Parameter Estimation of Superimposed Exponential Signals in Noise", IEEE Trans. on ASSP, 34(5):1081-1089, October 1986.

[3] P. Stoica and K. Sharman, "Maximum Likelihood Methods for Direction-of-Arrival Estimation", IEEE Trans. on ASSP, 38(7):1132-1143, July 1990.

[4] R. Roy and T. Kailath, "ESPRIT - Estimation of Signal Parameters via Rotational Invariance Techniques", IEEE Trans. on ASSP, 37(7):984-995, July 1989.

[5] C. Mathews and M. Zoltowski, "Eigenstructure Techniques for 2-D Angle Estimation with Uniform Circular Arrays", IEEE Trans. Sig. Proc., 42(9):2395-2407, Sept. 1994.

[6] A. van der Veen, P. Ober, and E. Deprettere, "Azimuth and Elevation Computation in High Resolution DOA Estimation", IEEE Trans. Sig. Proc., 40(7):18281832, July 1992.

[7] A. Swindlehurst and T. Kailath, "Azimuth/Elevation Direction Finding Using Regular Array Geometries", IEEE Trans. on AES, 29(1):145-156, January 1993.

[8] M. Clark and L. Scharf, "Two-Dimensional Modal Analysis Based on Maximum Likelihood", IEEE Trans. on Sig. Proc., 42(6):1443-1452, June 1994.

[9] M. Zoltowski, M. Haardt, and C. Mathews, "ClosedForm 2-D Angle Estimation with Rectangular Arrays in Element Space or Beamspace via Unitary ESPRIT", IEEE Trans. Sig. Proc., 44(2):316-328, Feb. 1996.

[10] A. Swindlehurst, B. Ottersten, R. Roy, and T. Kailath, "Multiple Invariance ESPRIT", IEEE Trans. on Sig. Proc., 40(4):867-881, April 1992.

[11] N. Sidiropoulos, R. Bro, and G. Giannakis, "Parallel Factor Analysis in Sensor Array Processing", IEEE Trans. Sig. Proc. (in review), 1999. 